\title{
X Congreso Ibérico de Agroingeniería

\section{Reescribir los retos del agua con una amplia base social. Grupo Operativo: Partenariado del Agua de la Cuenca del Ebro-Aragón}

\author{
Á. Jiménez Jiménez ${ }^{1}$, I. Oliván Lambea², J.R. López Pardo ${ }^{3}$, Y. Gimeno Cuenca ${ }^{4}$, V. Zapata \\ Ruiz $^{5}$, R. Castillo López ${ }^{6}$ \\ Colegio Oficial de Ingenieros Agrónomos de Aragón, Navarra y País Vasco; decano@coiaanpv.org \\ 2 Colegio Oficial de Ingenieros Agrónomos de Aragón, Navarra y País Vasco; \\ secretariotecnico@coiaanpv.org \\ 3 Colegio Oficial de Ingenieros Agrónomos de Aragón, Navarra y País Vasco \\ 4 Riegos del Alto Aragón; ygimeno@riegosaltoaragon.es \\ 5 EEAD-CSIC; v.zapata@csic.es \\ 6 CINGRAL; rcastillo@cingral.com
}

\begin{abstract}
Resumen: El Partenariado del Agua del Ebro-Aragón es un Proyecto que se está ejecutando por el Grupo Operativo del mismo nombre en el marco del Programa de Desarrollo Rural de Aragón 14-20. El estudio y recopilación de los aspectos técnicos, económicos y sociales del regadío generará una geodatabase que permitirá trabajar en tres laboratorios de innovación. El primer laboratorio de innovación abordará la búsqueda de casos de éxito y buenas prácticas, el segundo laboratorio el análisis y prospectiva de las infraestructuras de riego y el tercer taller se encargará de la comunicación interna y externa del Proyecto, sirviendo de canal de adquisición de información y de herramienta para la difusión de los resultados. El proyecto pretende reescribir los retos del agua desde una visión actualizada que aborde los retos del futuro. Será un esbozo de cómo debería evolucionar la gestión del agua, con una perspectiva técnica pero socialmente integradora y con la ambición de crear un modelo atractivo, en el que todos los ciudadanos del Ebro-Aragón quieran instalarse.
\end{abstract}

Palabras clave: agua, regadío, gobernanza, integración

\section{Introducción}

La Directiva Marco del Agua hace hincapié en el diálogo para superar las dificultades en la gestión del agua. Hace noventa años en el Ebro se puso en marcha de forma pionera en el mundo la gestión participativa de la cuenca hidrográfica. Los retos que trae el siglo XXI hacen pensar que éste es el momento de dar un nuevo impulso a este modelo.

La discusión acerca del uso del agua en el Ebro-Aragón se establece entre los términos técnicos y los políticos. Ambos son necesarios para identificar soluciones factibles, ampliamente aceptadas y que cumplan con los objetivos de la planificación pública.

Los aspectos técnicos tienen que tender puentes entre disciplinas que suelen vivir aisladas, como la hidrología, la ingeniería, la economía, la ecología, la sociología o el derecho. Técnica y política deben de combinarse para decidir qué bienes y servicios se van a aportar a la sociedad con el agua del Ebro.

El Partenariado del Agua del Ebro se creó informalmente en 2012, y documentalmente en abril de 2014. Se trata de una plataforma de cooperación para la promoción e impulso de un uso 
racional del agua como factor de desarrollo y equilibrio territorial, y de la protección del agua y del suelo como recursos naturales estratégicos de la cuenca del Ebro. El Partenariado presentó en mayo de 2017 una solicitud en respuesta a la convocatoria del Gobierno de Aragón de Grupos Operativos del PDR 2014-2020. La solicitud restringió el ámbito de actuación a la parte aragonesa de la Cuenca (el Ebro-Aragón).

El Grupo Operativo está coordinado por el Colegio Oficial de Ingenieros Agrónomos de Aragón, Navarra y País Vasco, que junto con la Comunidad general de regantes de Riegos del Altoaragón, el Colegio Oficial de Ingenieros Técnicos Agrícolas de Aragón y la Unión de Agricultores y Ganaderos de Aragón, son los socios beneficiarios de dicho grupo operativo. Es asimismo socia no beneficiaria, la Comunidad de regantes del Canal Imperial de Aragón.

Para la ejecución del proyecto se ha contratado a cuatro centros tecnológicos que se harán cargo de las partes que lo componen. Se trata del Departamento de Geografía y Ordenación del Territorio de la Universidad de Zaragoza, el grupo de investigación Riego, Agronomía y Medio Ambiente de la EEAD-CSIC, el Área de Información y Documentación del CITA y la Asociación Española de Periodistas Agroalimentarios.

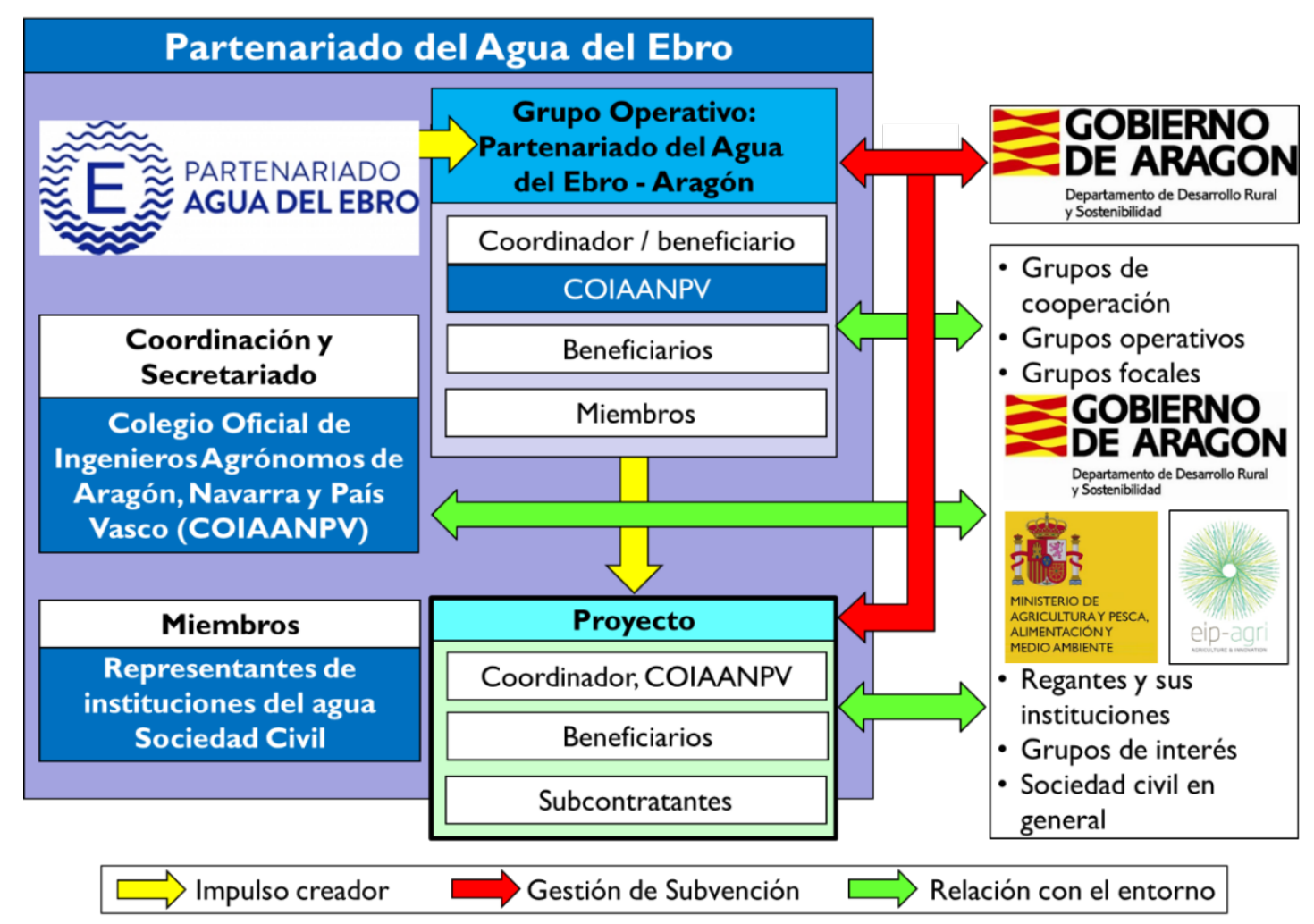

Figura 1. El Partenariado del Agua del Ebro, el Grupo Operativo y el Proyecto de Cooperación. Liderazgo, Miembros, relaciones entre ellos y con otros elementos del entorno.

\section{Estructura del Proyecto}

Tal y como se representa en la Figura 2, el proyecto se estructura en dos objetivos:

- Objetivo 1: Análisis del regadio en el Ebro-Aragón

- Objetivo 2: Laboratorios de Innovación 


\section{Objetivo I:Análisis del regadío en el Ebro-Aragón}

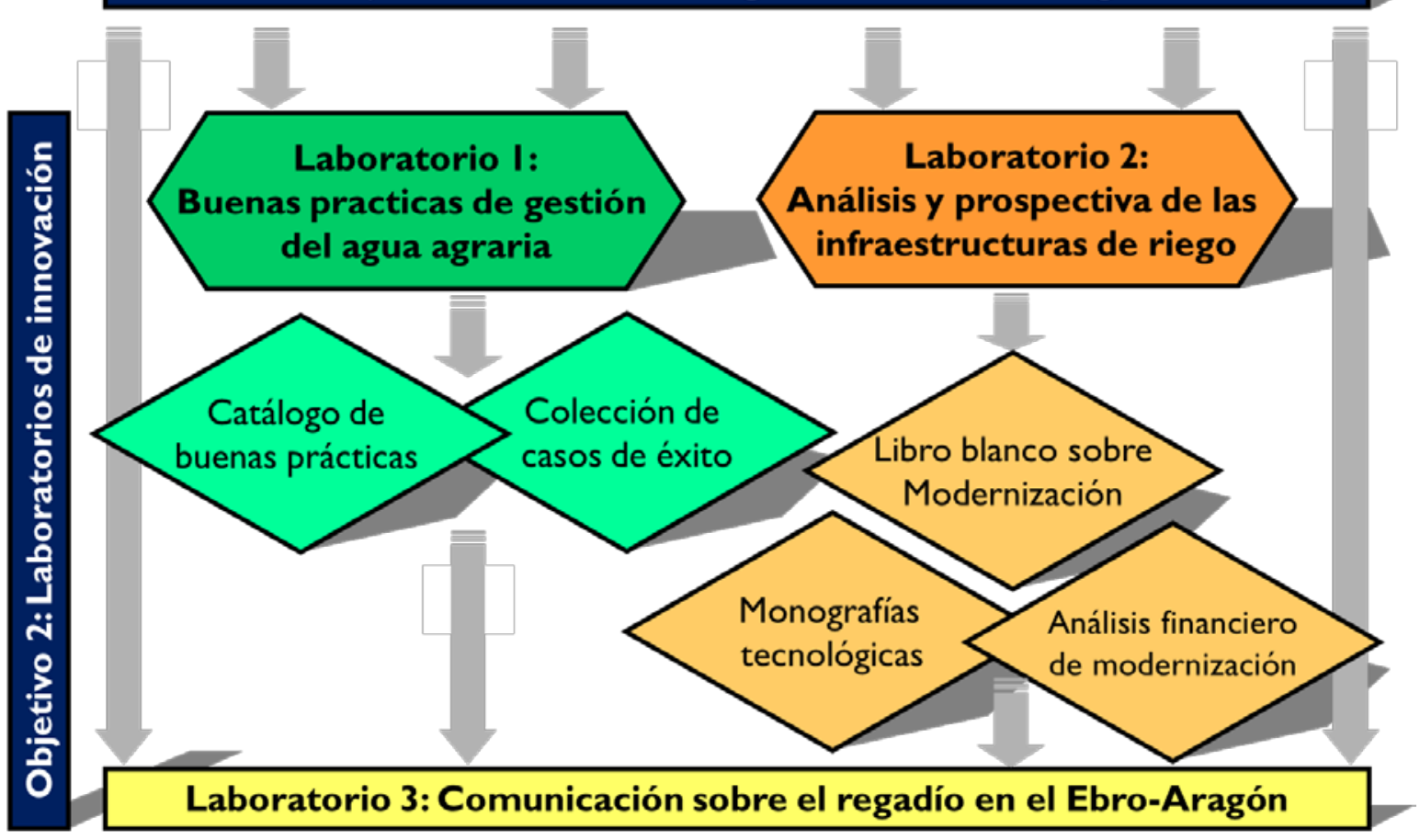

Figura 2. Diagrama funcional de objetivos, laboratorios y resultados principales.

El Objetivo 1 pretende actualizar el mapa del aprovechamiento del agua en Aragón mediante la creación de una geodatabase que contenga la situación de las zonas regables, su potencialidad, su socio economía, sus rasgos hidrológicos y su valor estratégico para el desarrollo regional y local. Analizar los datos de la situación actual con amplitud de miras, para progresar hacia una planificación estratégica de las distintas tipologías de zonas regables. Generar una imagen del regadío diversificada, que sirva de nicho para la generación de iniciativas plurales.

Se pretende obtener la actualización del análisis del regadío en Aragón, generando el mapa de aprovechamiento del agua atendiendo a su situación, potencialidad, valor estratégico para el desarrollo regional y local.

La recopilación de toda esa información será plasmada sobre un medio informático accesible, que permitirá a los usuarios interaccionar y que será la base del análisis para el Proyecto. Se obtendrá una geodatabase con información de tipo geográfico, socioeconómico, hidrológico y ambiental del regadío. Asimismo, esta base cartográfica servirá para integrar los resultados del Proyecto, asociándolos a su localización.

Por otro lado, la geodatabase debe proporcionar soporte cartográfico al laboratorio 3, facilitando la labor de diseminación del conocimiento generado en el proyecto. 

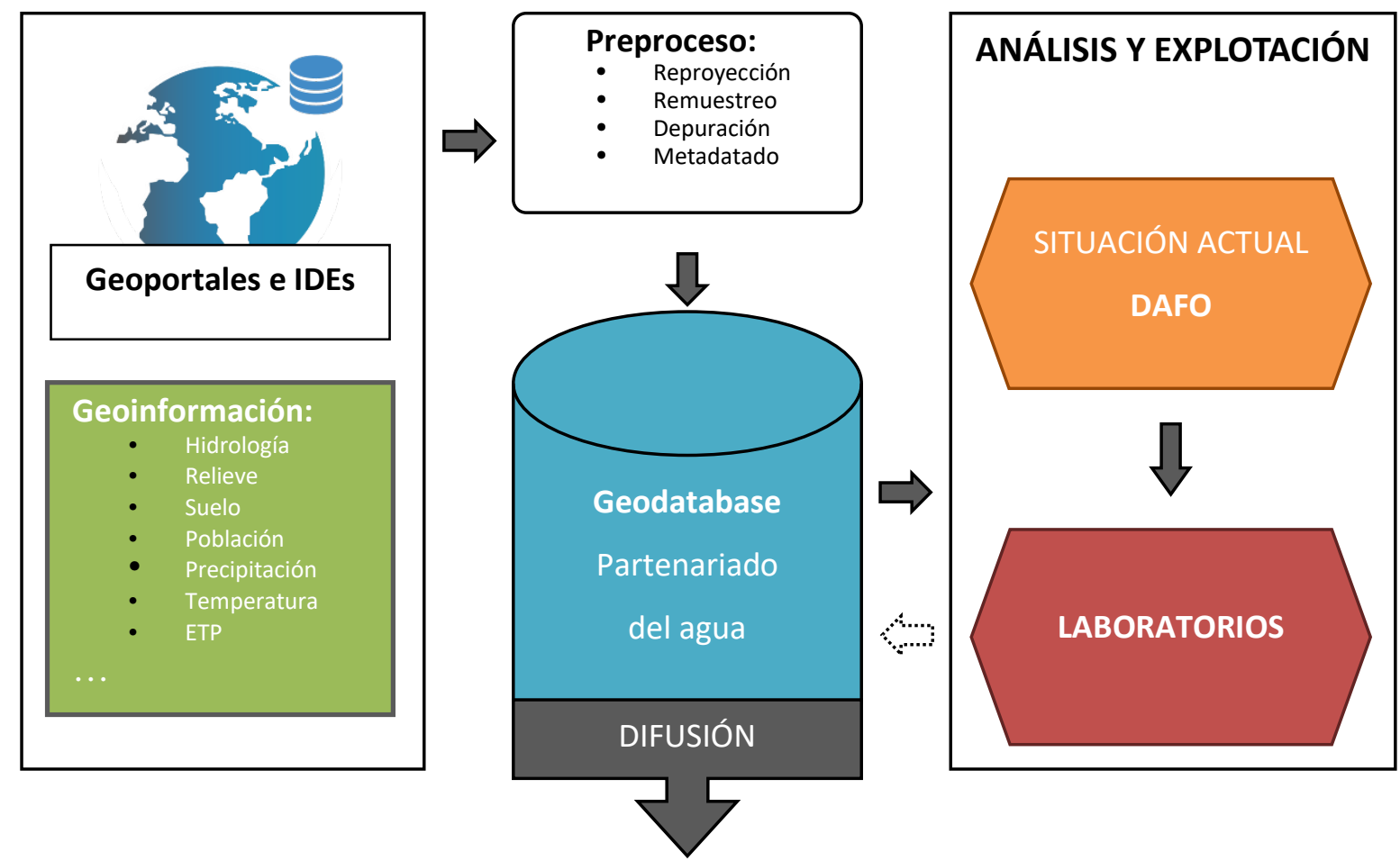

Figura 3. Diagrama de flujos en relación con el Laboratorio 1, mostrando las fuentes de información y las tareas de análisis, así como su relación con los laboratorios 2 y 3.

El objetivo 2 será desarrollado por tres laboratorios:

- Laboratorio 1: búsqueda de casos de éxito y buenas prácticas de gestión del agua agraria.

- Laboratorio 2: análisis y prospectiva de las infraestructuras de riego en Aragón.

- Laboratorio 3: comunicación sobre el regadío en el Ebro-Aragón.

El Laboratorio 1 se encargará del análisis de la "parte inmaterial del agua", mediante la aplicación de técnicas de gestión del conocimiento para la identificación de "buenas prácticas" (BP) y de "casos de éxito" (CE) relativos a la gestión del agua agrícola en el Valle del Ebro. Este conocimiento surge tanto desde los centros de innovación (públicos o privados) como desde los propios usuarios. La estructuración de este conocimiento permitirá poner en valor mediante herramientas de difusión y divulgación tanto las prácticas existentes de gestión, como de cooperación entre los diferentes usuarios, como aquellas que se pudieran poner en marcha en un futuro. Para lograr este objetivo se ha diseñado una herramienta de participación a través de las redes sociales.

En el Laboratorio 2 se va a analizar la "parte material del riego", su planificación, la evolución de las estructuras de regadío en el Ebro-Aragón, su estado de avance tecnológico y los retos que este representa y como se vincula y armoniza con las necesidades productivas.

Se realizará el análisis de la situación actual y el estudio de las infraestructuras de regadío y sus inversiones, ayudará a definir una línea de planificación estratégica de las distintas zonas regables.

El resultado final producirá una colección de monografías técnicas y una serie de modelos económico-financieros aplicables a las modernizaciones de los diferentes tipos de regadíos identificados y un libro blanco de la modernización de regadíos en el EBRO-Aragón.

Laboratorio 3: la comunicación va a ser uno de los elementos clave en el proyecto. Por un lado, se diseminará todo el conocimiento generado y recopilado en el Grupo y por otro, se tratará de comunicar experiencias cotidianas, dirigidas particularmente a la población urbana. 


\section{CONGRESO IBÉRICO DE AGROINGENIERÍA \\ X CONGRESSO IBÉRICO DE AGROENGENHARIA \\ 3 - 6 septiembre 2019, Huesca - España}

Dentro de estas experiencias, se abordarán los aspectos positivos del agua sobre la vertebración del territorio, así como también la relación entre agua y producción de alimentos y mantenimiento del paisaje del medio rural. El laboratorio se basa en tres ejes de actuación:

- Comunicación interna: destinada a la difusión de los materiales de trabajo entre los socios.

- Comunicación externa: que se establece con los destinatarios directos y los potenciales beneficiarios de los resultados obtenidos.

- Difusión general: con el público en general, a través de prensa y redes sociales.

\section{Resultados y discusión}

Los resultados previstos se pueden clasificar en los siguientes ámbitos:

Eficiencia en el uso de los recursos naturales. El proyecto guarda una estrecha relación con la modernización de los regadíos de Aragón, que se ha desarrollado fundamentalmente en las dos primeras décadas del siglo XXI. En la medida en que el proyecto impulsará el buen uso del agua en el regadío, facilitará la transición en la eficiencia de riego desde valores próximos al 50\% hasta valores próximos al 90\%[1], según los análisis realizados en Aragón. Esta transición que se viene realizando en las comunidades que modernizan sus estructuras y su gestión - se verá apoyada por los resultados del proyecto. Así, se analizará el futuro de estas políticas y la modernización de tipos y zonas de regadíos que hasta ahora no se han beneficiado de proyectos de inversión público-privada.

Desarrollo rural sostenible en el ámbito del regadío. El proyecto analizará indicadores socioeconómicos de los regadíos relacionados con la renta, el empleo y la demografía. El objetivo es contribuir al desarrollo rural sostenible, identificando y promoviendo alternativas estructurales y de gestión que redunden en una mejora de estos indicadores. La falta de un análisis cuantitativo de la situación actual y el corto periodo de tiempo de duración del proyecto en comparación con el tiempo requerido para la mejora de las infraestructuras y la gestión del regadío hacen que no sea posible en este momento cuantificar la mejoría esperable en estos indicadores.

Viabilidad económica de las explotaciones de regadío. Los análisis económicos de los procesos de modernización de regadíos realizados en Aragón hasta la fecha, muestran una mejoría en los resultados económicos de las explotaciones. Las directrices para la mejora futura de las infraestructuras y de la gestión del riego permitirán seguir en esta dirección, poniendo énfasis en aspectos como la reducción de la factura energética, la optimización del coste de las redes colectivas, o la adecuación de las prestaciones de telecontrol a las capacidades de gestión y mantenimiento de cada comunidad de regantes. En el futuro, los proyectos de modernización de regadíos deberían de huir de la "talla única", para ajustarse dinámicamente a cada caso particular, a su realidad económica, social y técnica.

Adaptación y mitigación del cambio climático. La modernización de los regadíos tiene muchas conexiones con la adaptación y la mitigación del cambio climático, pero sólo algunas de ellas están claramente establecidas. Entre las positivas se cuenta la reducción en el uso del agua (cuantificada localmente en un 30\%[2]), o la disminución de la contaminación por nitratos (medida en un $76 \%$ en una comunidad de regantes de Aragón). El aspecto que hasta la fecha ha resultado más negativo es el aumento de las necesidades de energía para presurizar el agua. Así, entre 1970 y 2007 el consumo energético del regadío español aumentó un 657\% (de 206 a $1.560 \mathrm{kWh} / \mathrm{ha}$, Corominas, 2010[3]). El proyecto propondrá acciones estructurales y de manejo que permitan profundizar en la reducción del uso del agua y de la contaminación, así como revertir la tendencia en cuanto al consumo de energía para bombeos.

Soporte a políticas innovadoras sobre regadíos. El equipo del proyecto espera que éste sea uno de los resultados más importantes del proyecto. Las actividades de prospectiva y de elaboración de amplios consensos sociales permitirán hacer una contribución a la formulación 


\section{CONGRESO IBÉRICO DE AGROINGENIERÍA \\ X CONGRESSO IBÉRICO DE AGROENGENHARIA \\ 3 - 6 septiembre 2019, Huesca - España}

de políticas de regadíos que aseguren su sostenibilidad social, económica y ambiental. Así, desde el proyecto se pretende dar respuesta a preguntas del tipo:

¿Cómo fortalecer la gobernanza de las comunidades de regantes?

¿Cómo planificar la modernización de las zonas regables para minimizar las necesidades energéticas?

¿Cómo aprovechar las energías renovables en el contexto de futuras regulaciones de la generación y la conexión a la red?

¿Cómo gestionar los regadíos para minimizar la contaminación agraria y para consumir los nutrientes que contaminan el agua de riego en la producción agraria?

¿Qué hacer con los regadíos de ribera o de zonas templadas, en los que la modernización casi no ha entrado hasta la fecha?

¿Cómo minimizar el coste de las obras de modernización de regadíos en diferentes contextos geográficos y orientaciones productivas?

¿Cómo diseñar y gestionar las redes para hacer frente a problemas emergentes, como escasez, especies invasoras o cambio climático?

¿Cómo usar mejor los fondos públicos para fomentar la transformación del regadío del Ebro aragonés, fomentando la productividad y la sostenibilidad?

Impacto social en las zonas regables y en la sociedad en general. El proyecto pretende crear una transformación cualitativa (de difícil cuantificación) en la percepción del regadío del Ebro-Aragón tanto en las zonas regables como en la sociedad general. El equipo del proyecto quiere proyectar su visión hacia el futuro para contribuir a fomentar el posibilismo, la proactividad y la gobernanza del sector.

En la figura 4 se representa el diagrama de los flujos alrededor del Grupo Operativo y la interacción con otros grupos de cooperación sectoriales, así como con las instituciones y administraciones involucradas en la gobernanza del agua. 


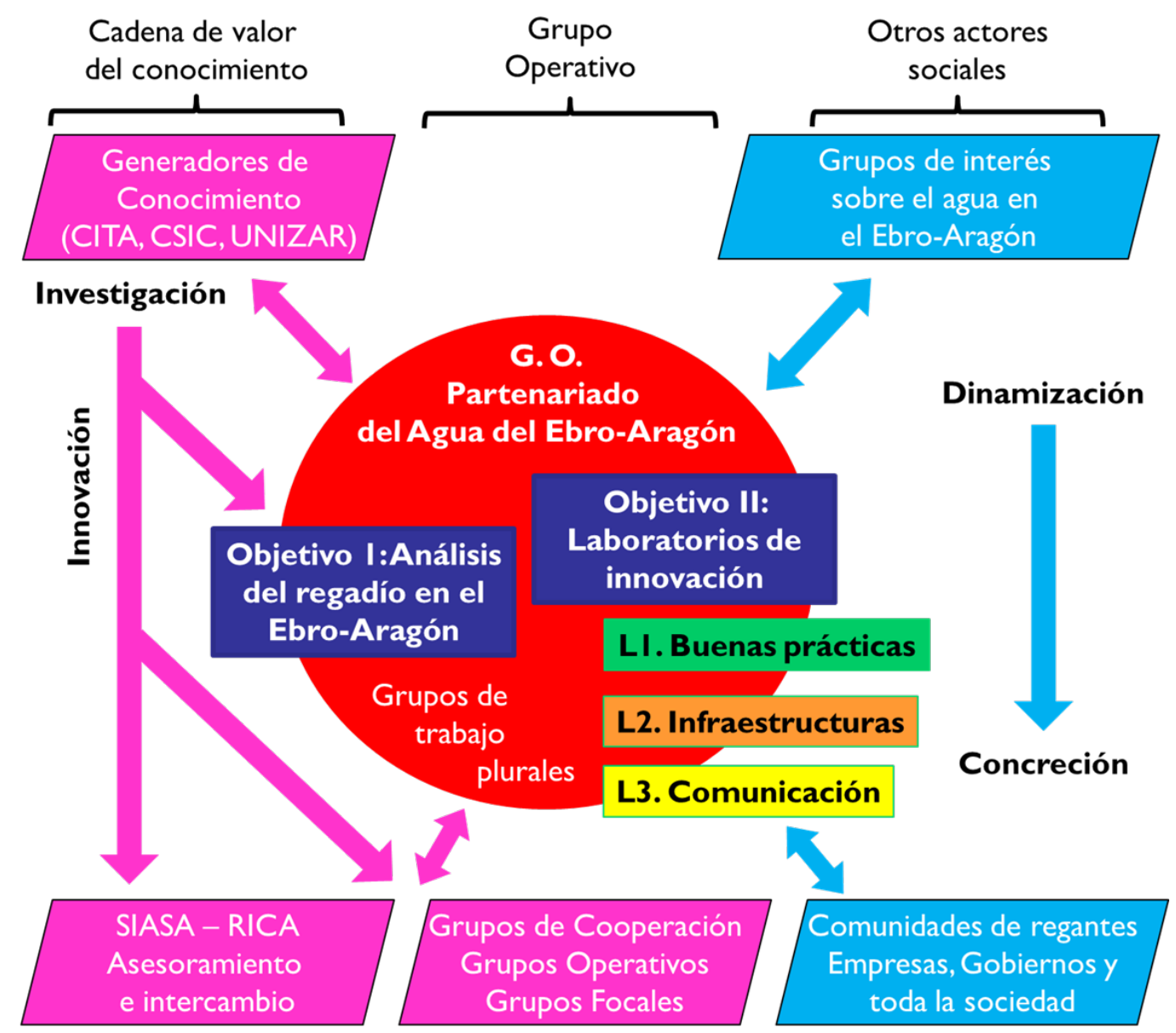

Figura 4. Diagrama de los flujos alrededor del Grupo Operativo y su proyecto. Se representan los flujos relativos a la cadena de valor del conocimiento, los flujos que van de la dinamización a la concreción, y los aspectos de divulgación y comunicación en el marco del proyecto (derivados del Laboratorio 3).

\section{Conclusiones}

El Grupo Operativo cree firmemente en la capacidad local para generar acuerdos y en la ventaja de la gestión participativa y quiere crear un estado de opinión sobre el regadío, basado en los conocimientos recopilados y creados en este proyecto.

En definitiva el proyecto pretende proporcionar argumentos a las administraciones para ayudarles en la toma de decisiones, en la planificación estratégica, y por lo tanto en su política de regadíos. Además, se pretende divulgar en la sociedad que la realidad del regadío del EbroAragón va más allá de conflictos, desencuentros o desastres naturales.

\section{Referencias}

1. Jiménez-Aguirre, M.T., Isidoro, D. 2018. Hydrosaline Balance in and Nitrogen Loads from an irrigation district beforeand after modernization. Agric. Water Manage. 2018, 208 (63-175).

2. Lecina, S., Isidoro, D., Playán, E., Aragüés, R., 2010. Irrigation Modernization in Spain: Effects on Water Quantity and Quality: A conceptual approach. International Journal of Water Resources Development. 2010, 26(2) (265-282)

3. Corominas, J. Agua y energía en el riego, en la época de la sostenibilidad. Ingeniería del agua. 2010, 17(3), (219-233). 